\title{
Real-time detection of antibiotic activity by measuring nanometer-scale bacterial deformation
}

\author{
Rafael Iriya \\ Karan Syal \\ Wenwen Jing \\ Manni Mo \\ Hui Yu \\ Shelley E. Haydel \\ Shaopeng Wang \\ Nongjian Tao
}




\title{
Real-time detection of antibiotic activity by measuring nanometer-scale bacterial deformation
}

\author{
Rafael Iriya, ${ }^{a, b}$ Karan Syal, ${ }^{a}$ Wenwen Jing, ${ }^{a}$ Manni Mo, ${ }^{a}$ Hui Yu, ${ }^{a, c}$ Shelley E. Haydel, ${ }^{d, e}$ Shaopeng Wang, ${ }^{a, c}$ and \\ Nongjian Tao ${ }^{a, b, c, *}$ \\ ${ }^{a}$ Arizona State University, Biodesign Center for Biosensors and Bioelectronics, Tempe, Arizona, United States \\ ${ }^{\mathrm{b}}$ Arizona State University, School of Electrical, Computer and Energy Engineering, Tempe, Arizona, United States \\ 'Nanjing University, State Key Laboratory of Analytical Chemistry for Life Science, School of Chemistry and Chemical Engineering, Nanjing, China \\ ${ }^{\mathrm{d} A r i z o n a}$ State University, School of Life Sciences, Tempe, Arizona, United States \\ ${ }^{\text {e} A r i z o n a}$ State University, Biodesign Institute Center for Immunotherapy, Vaccines and Virotherapy, Tempe, Arizona, United States
}

\begin{abstract}
Diagnosing antibiotic-resistant bacteria currently requires sensitive detection of phenotypic changes associated with antibiotic action on bacteria. Here, we present an optical imaging-based approach to quantify bacterial membrane deformation as a phenotypic feature in real-time with a nanometer scale $(\sim 9 \mathrm{~nm})$ detection limit. Using this approach, we found two types of antibiotic-induced membrane deformations in different bacterial strains: polymyxin B induced relatively uniform spatial deformation of Escherichia coli O157:H7 cells leading to change in cellular volume and ampicillin-induced localized spatial deformation leading to the formation of bulges or protrusions on uropathogenic E. coli CFT073 cells. We anticipate that the approach will contribute to understanding of antibiotic phenotypic effects on bacteria with a potential for applications in rapid antibiotic susceptibility testing. ( $) 2017$ Society of Photo-Optical Instrumentation Engineers (SPIE) [DOI: 10.1117/1.JBO.22.12.126002]
\end{abstract}

Keywords: cell membrane deformation; Escherichia coli; antibiotics; polymyxin; ampicillin.

Paper 170422RR received Jul. 5, 2017; accepted for publication Nov. 17, 2017; published online Dec. 12, 2017.

\section{Introduction}

Antibiotic resistance poses serious threats to humans with multidrug-resistant bacteria now commonly known as "superbugs."1-4 To combat antibiotic-resistant bacteria, one must develop more effective and rapid antibiotic susceptibility testing (AST) methods. ${ }^{5}$ Current AST technologies used in clinic labs rely on cell culture, which can take 1 to 14 days, depending on the growth rate of the bacteria. ${ }^{6,7}$ A critical task in AST is to quickly detect if an antibiotic at a given concentration has a phenotypic effect on the bacteria. One commonly monitored phenotypic feature is the bacterial cell morphology visualized via optical microscopy. ${ }^{8}$ Other phenotypic features explored for rapid AST include bacterial nanomotion associated bacterial metabolic activities, ${ }^{9}$ measuring bacterial growth with magnetic bead rotation sensors, ${ }^{10}$ nanometer (nm)-scaled bacterial cell motions measured using atomic force microscopy, ${ }^{11}$ changes in plasmonic imaging responses, ${ }^{12}$ and detection of chemical signatures with electrochemical methods. ${ }^{13}$

In the present work, we report an optical method to track $\mathrm{nm}$-scale membrane deformations in individual bacterial cells with the goal to detect antibiotic action at the earliest possible stage. The method detects and quantifies subtle bacterial membrane deformations that are difficult to detect using traditional optical imaging analysis methods. ${ }^{14,15}$ We study the membrane deformations of two different Escherichia coli strains, E. coli O157:H7 and uropathogenic E. coli CFT073, ${ }^{16}$ associated with antibiotics, polymyxin $\mathrm{B}(\mathrm{PMB})$ and ampicillin, respectively.

\section{Materials and Methods}

\subsection{Overview}

E. coli $\mathrm{O} 157: \mathrm{H} 7$ and uropathogenic E. coli (UPEC) strain CFT073 cultures were prepared by diluting overnight cultures into fresh Luria broth (LB) and continuing growth for $2 \mathrm{~h}$ at $37^{\circ} \mathrm{C}$ with gentle rotary mixing until the cells reached the midexponential growth phase. The individual bacterial cells were tethered on a glass slide via antibody coupling. For antibiotic testing, bacterial cells were incubated in LB or phosphatebuffered saline (PBS) prior to the addition of ampicillin or $\mathrm{PMB}$, respectively. Optical images of the antibiotic effects on the bacteria were obtained with an inverted optical microscope with a $100 \times$ objective (numerical aperture of 1.49) and recorded with a CCD camera [Fig. 1(a)]. A key task of our method is to track local cellular membrane deformation, including expansion and contraction, associated with antibiotic action. We describe the local membrane deformation with displacement vectors [Fig. 1(b)]. A displacement vector points in the direction normal to the local cell edge with magnitude representing the amount of deformation.

\subsection{Differential Detection Method}

To track subtle cellular deformation, we used a differential detection method, ${ }^{17}$ which can track deformations as small as $\sim 9 \mathrm{~nm}$ (described in the Sec. 3). The method establishes a linear relation between cell deformation and the intensity profile of a region of interest (ROI) that includes the cell edge. The ROI is divided into two halves with one half inside and the other half outside of the cell. The total pixel intensities of 
(a)

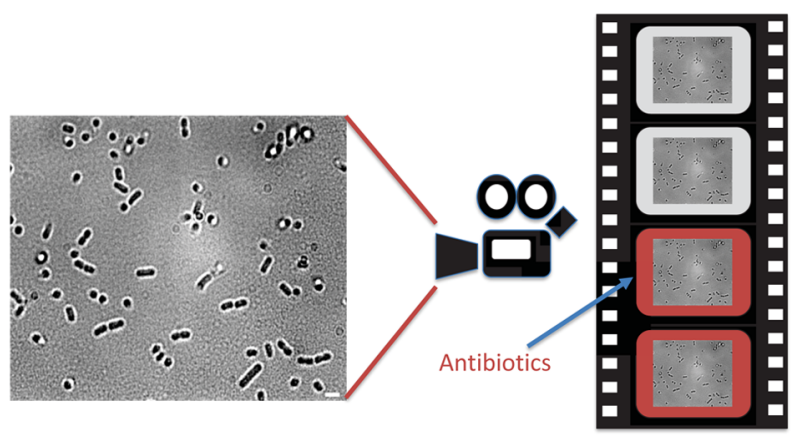

(b)

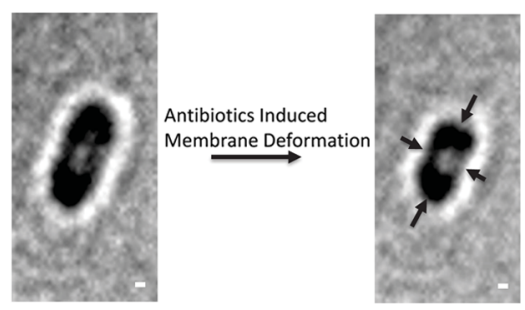

Fig. 1 Nanometer-scale optical tracking of bacterial cell deformation. (a) Bacterial cells (E. coli O157:H7) were imaged by an optical microscope and their responses to antibiotics are recorded and analyzed. Scale bar: $2 \mu \mathrm{m}$. (b) Antibiotic-induced bacterial membrane deformation (shape, length, and width) described by displacement vectors (black arrows). Scale bar: $200 \mathrm{~nm}$.

these two halves are denoted by $I_{1}$ and $I_{2}$, respectively [Fig. 2(a)]. When the cell expands, $I_{1}$ decreases and $I_{2}$ increases. In contrast, when the cell shrinks, $I_{1}$ increases and $I_{2}$ decreases. The edge displacement $d$ is given by

$d=k \frac{I_{2}-I_{1}}{I_{1}+I_{2}}$

where $k$ is a constant obtained through a calibration process. The calibration is performed in the first image frame by moving the ROI normal to the cell edge [Fig. 2(b)]. The differential intensity change versus the ROI movement in terms of pixels reveals a linear relation within a certain range of pixel shift [magnified region of Fig. 2(b)]. To obtain a smooth curve and achieve subpixel sensitivity, the images are interpolated by 5 pixels (i.e., 4 pixels inserted in between two adjacent pixels in both $x$ - and $y$-directions).

The linear regime is typically within 4 pixels (or 20 pixels after interpolation), which corresponds to an actual cellular deformation range of $300 \mathrm{~nm}$.

\subsection{Materials}

E. coli O157:H7 (ATCC 43888) and UPEC CFT073 were purchased from Fisher Scientific and ATCC, respectively. Affinity-purified goat anti-E. coli $\mathrm{O} 157: \mathrm{H} 7 \mathrm{IgG}$ polyclonal antibodies, purchased from Kirkegaard and Perry Laboratory, Inc. (Gaithersburg, Maryland), were added to 1-mL PBS (1×) and stored at $-20^{\circ} \mathrm{C}$ prior to use. PMB and ampicillin were purchased from Sigma-Aldrich, dissolved in PBS (1×) at stock concentrations of 10 and $2 \mathrm{mg} / \mathrm{mL}$, respectively, and stored in dark at $2^{\circ} \mathrm{C}$ to $8^{\circ} \mathrm{C}$. 3-Triethoxysilylpropylamine $99 \%$ (APTES), N-Hydroxysuccinimide $98 \%$ (NHS), $N$-Ethyl- $N^{\prime}$-(3-dimethylaminopropyl) carbodiimide 97\% (EDC), and sodium acetate $(\mathrm{NaOAc})$ were also purchased from Sigma-Aldrich. (a)

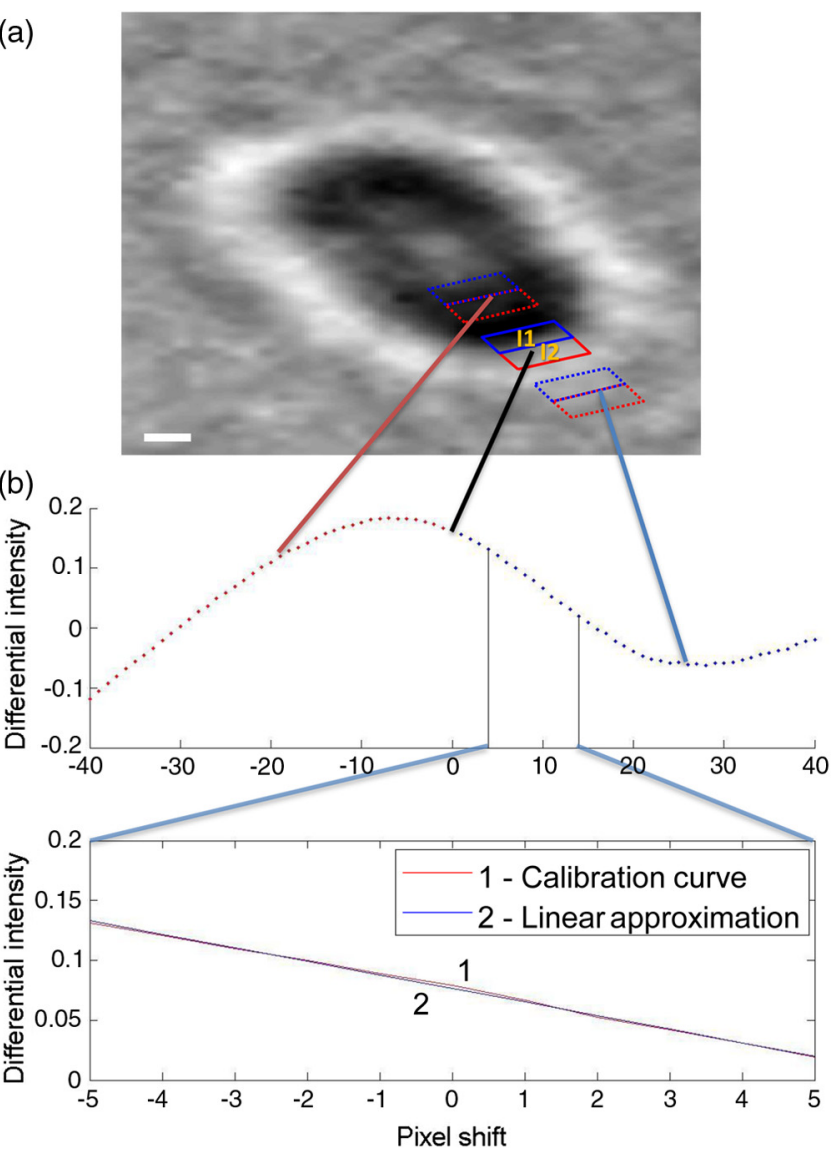

Fig. 2 Principle and calibration of the nanometer-scale optical tracking method. (a) An $\mathrm{ROI}$ at the cell edge was selected (bluered rectangle) and divided into two halves (blue and red) with optical intensities denoted by $I_{1}$ and $I_{2}$, respectively. Differential intensity, $\left(I_{2}-I_{1}\right) /\left(I_{1}+I_{2}\right)$, provided sensitive measurement of the local cell membrane deformation. To calibrate the method, the ROI was shifted (orthogonal to the cell edge) by known number of pixels (pixel shift), and differential intensity was determined. Scale bar: $200 \mathrm{~nm}$. (b) Differential intensity versus pixel shift, where a region of $\sim 10$ pixels $(\sim 750 \mathrm{~nm})$ is linear, allowed precise determination of local cell deformation. 1 pixel (without interpolation) $=75 \mathrm{~nm}$.

\subsection{Bacterial Preparation}

All bacterial stocks were maintained in $5 \%$ glycerol at $-80^{\circ} \mathrm{C}$ in small aliquots $(20 \mu \mathrm{L})$. After overnight incubation at $37^{\circ} \mathrm{C}, E$. coli cells were diluted into fresh $\mathrm{LB}$ and grown for $\sim 2 \mathrm{~h}$ at $37^{\circ} \mathrm{C}$ with gentle rotary mixing until the cells reached the midexponential growth phase. Bacterial cells were pelleted via centrifugation at $2000 \mathrm{~g}$ for $30 \mathrm{~min}$ and resuspended in $1 \mathrm{~mL}$ of PBS (1x).

\subsection{Surface Preparation}

Glass coverslips $(22 \times 22 \mathrm{~mm})$ were cleaned with deionized water (DW) and ethanol and then dried with nitrogen gas. A small aliquot of $1 \%$ of APTES in 5\% ethanol was applied to the glass slides and allowed to dry at room temperature for 50 to $60 \mathrm{~min}$. After the slides were cleaned and dried, a mixture of $0.1 \mathrm{M}$ NHS and $0.4 \mathrm{M} \mathrm{EDC}$ in $1 \mathrm{ml}$ of DW was applied to the slides to produce NHS ester receptors. After 10-min incubation, the NHS/EDC slides were again cleaned thoroughly with water and ethanol. The NHS/EDC-activated surface was 
blow dried with nitrogen. After the addition of $30 \mu \mathrm{g} / \mathrm{mL}$ of antibody in 20-mM sodium acetate, $\mathrm{pH} 5.5$ to the slides for $60 \mathrm{~min}$, the slides were cleaned again thoroughly with water and ethanol followed by blow drying with nitrogen, generating an antibody-activated surface for imaging experiments.

\subsection{Optical Imaging System}

The optical imaging system included an Olympus IX-81 inverted microscope with a $100 \times$ oil immersion and a Pike 032B CCD camera (Allied Vision Technologies, Newbuyport, Massachusetts) to record videos, typically at 50 frames per second (fps) for the PMB experiments and 11.8 fps for the ampicillin experiments. A fast frame rate was used for the PMB experiments to maximize data collection. Since the ampicillin experiments required long video recording to capture bacterial cell bulge/protrusion events, a slower frame rate was used to reduce the data size.

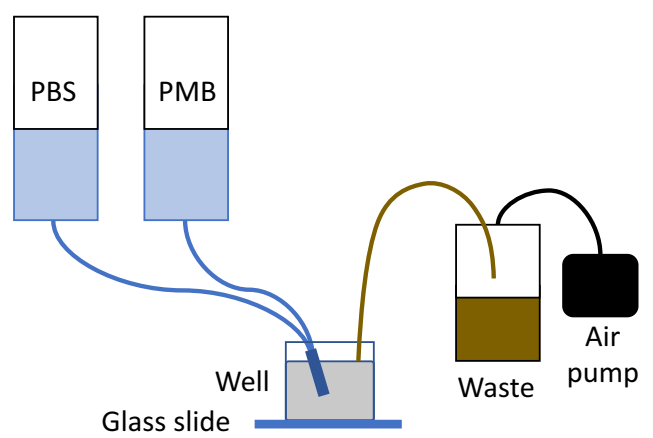

Fig. 3 Schematic for the drug perfusion system. As PBS or PMB was injected into the well, the suction pump concomitantly removed excess liquid from the well, thereby maintaining a constant solution volume.

\subsection{Bacterial Immobilization}

flexiPERM $^{\circledR}$ (Sarstedt Inc.) reusable wells $(0.5 \mathrm{~mL})$ were mounted and filled with $1 \times$ PBS or LB for the PMB or ampicillin experiments, respectively, and $10 \mu \mathrm{L}$ of bacterial cells $\left(\sim 10^{8}\right.$ cells). After 30 -min incubation at $37^{\circ} \mathrm{C}$ to allow bacterial binding to the surface-attached antibodies, unattached bacteria were removed using the flow setup (described below).

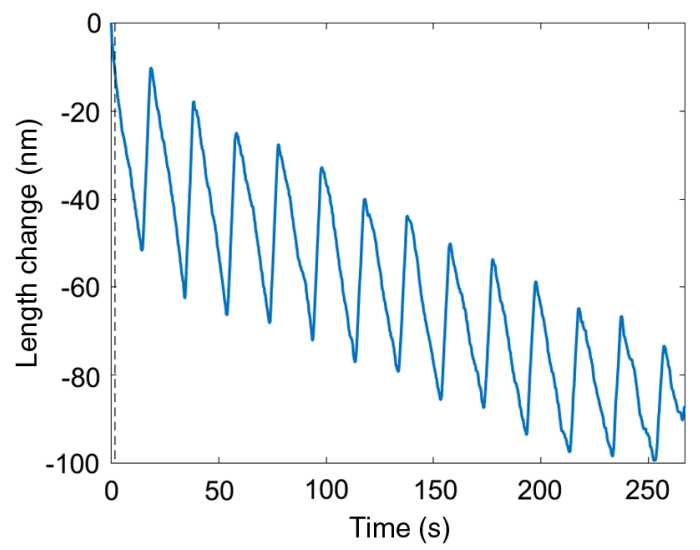

Fig. 5 Length change for a silicon bead when submitted to a $50 \mathrm{mHz}$ and $500 \mathrm{~nm}$ peak-to-peak modulation of the stage position in the $z$ direction (vertical), creating controlled focus changes. The modulation was realized by a piezo microscope stage (Physik Instrumente L.P., Auburn, Massachusetts, model P-545.3C7). From this experiment, there was evidence of long-term decay, due to stage drift, and an oscillation that responds nearly linearly to the modulation, with a peak of $\sim 25 \mathrm{~nm}$. These results indicated that a 10-nm stage change would correspond to a $1-\mathrm{nm}(250 / 25=10)$ measured length change. While short-term measurement windows would not be significantly affected, stage drift could affect interpretation of long-term measurement results. (a)
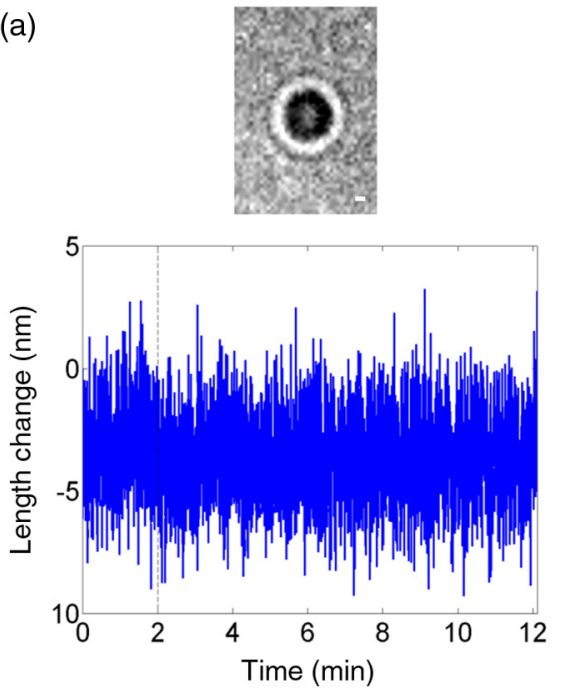

(b)
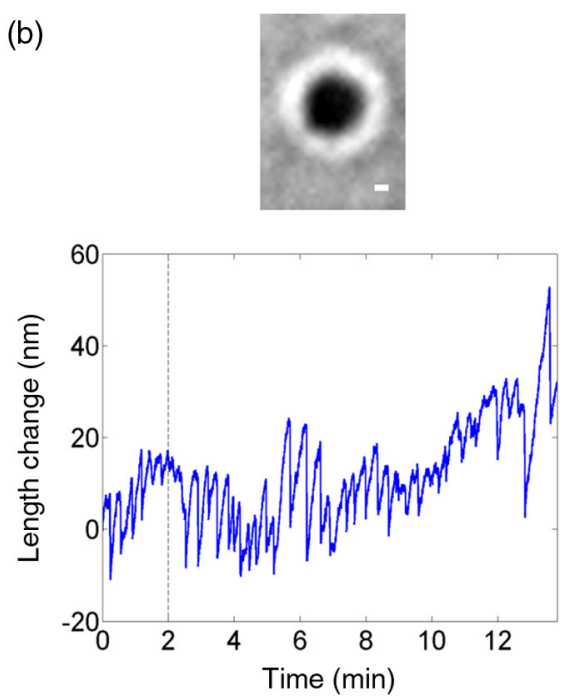

Fig. 4 Length change results for noise analysis. (a) A dust spot in the camera was selected to assess the noise of the algorithm without external factors. The signal was pure noise with standard deviation $\sigma=1: 66 \mathrm{~nm}$. (b) A silicon bead of $1 \mu \mathrm{m}$ was placed on the chip surface. Scale bar: $200 \mathrm{~nm}$. The silicon bead showed some small fluctuations and long-term noise, caused by external factors, such as small focus changes due to instabilities of the microscope stage. The short-term noise for a window of $10 \mathrm{~s}$ showed $\sigma=3 \mathrm{~nm}$. The limit of detection was $\sim 3 \sigma=9 \mathrm{~nm}$. 


\subsection{Flow Setup}

LB, $1 \times$ PBS, and PMB (diluted in PBS) were added to the wells through a gravity-based multichannel drug perfusion system with the excess liquid removed by an air pump. A schematic for the flow setup is shown in Fig. 3. The drug perfusion system delivered solutions at a flow rate of $330 \mu \mathrm{L} / \mathrm{min}$ with the transition time between different flow solutions ranging from 1 to $2 \mathrm{~s}$. For the ampicillin experiment, the antibiotics were manually pipetted into the mounted reusable wells atop the antibody surface with immobilized bacteria.

\subsection{Image Collection and Processing}

All image sequences were collected in transmitted mode at a pixel resolution of $640 \times 480$. We chose an appropriate exposure time to maximize image intensity and avoid over exposure.

\subsection{Data Analysis from Images}

Images were processed using custom-written MATLAB programs and the methods described previously in the paper. (a)

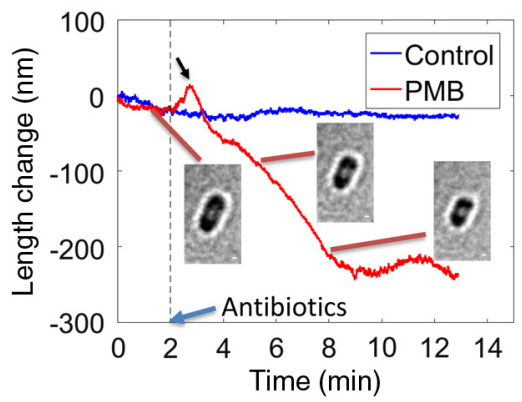

(b)

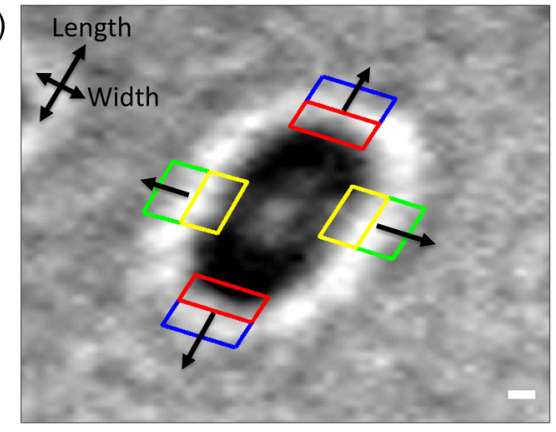

Fig. 6 (a) Cell length change of a bacterium (E. coli O157:H7) exposed to PMB $(0.5 \mu \mathrm{g} / \mathrm{mL}$ ) (red line) compared with PBS (blue line) over time. The arrow indicates the initial expansion shortly after antibiotic introduction. (b) Examples of ROls and the corresponding displacement vectors for the ROls. Length changes were determined by summing the two opposite vectors of the red/blue ROls, and width changes were determined by summing the opposite vectors of the green/yellow ROls. Scale bar: $200 \mathrm{~nm}$.

(a)
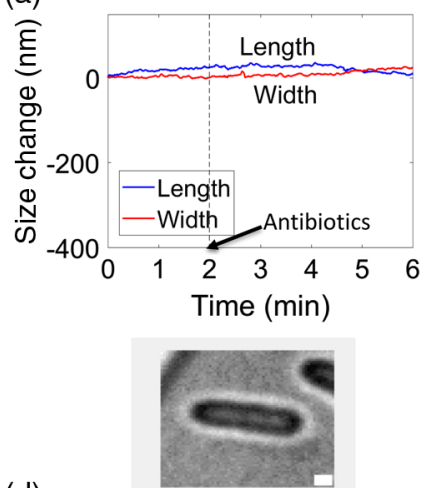

(d)

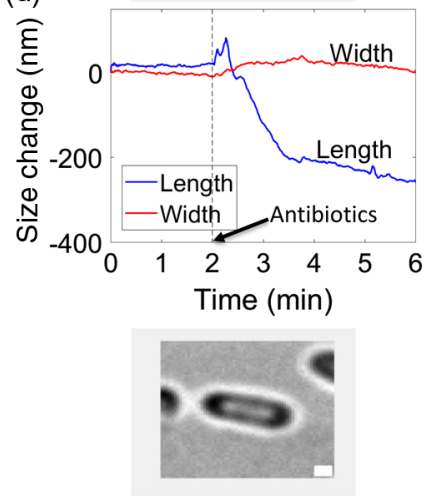

(b)
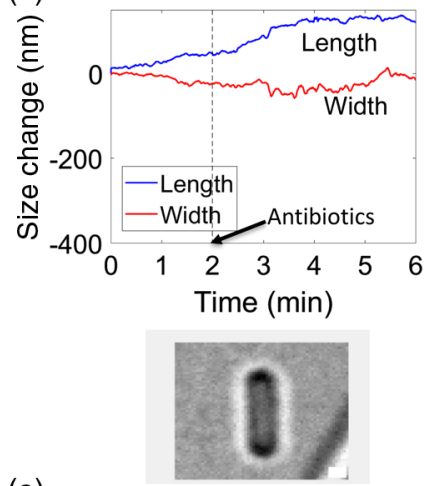

(e)

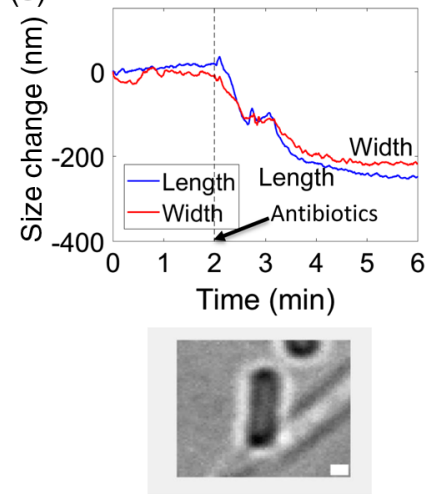

(c)
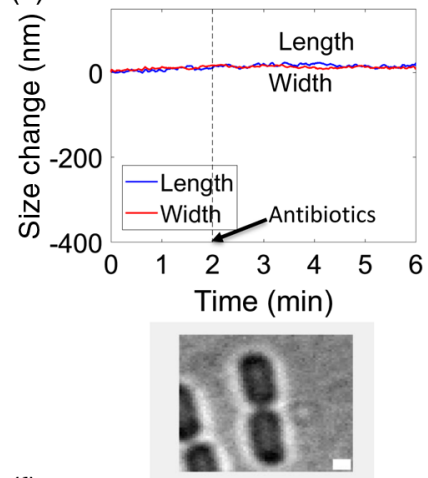

(f)

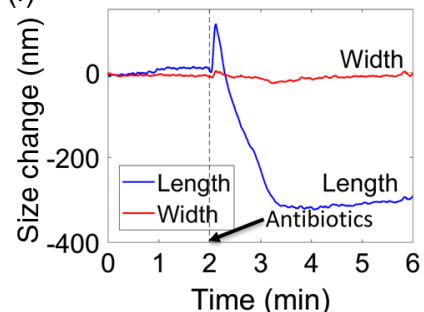

Time $(\min )$

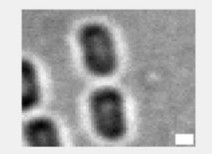

Fig. 7 Cell length (blue) and width (red) changes for three E. coli O157:H7 cells. (a-c) Three different cells incubated in PBS buffer only. (d-f) Three different cells exposed to PMB $(0.5 \mu \mathrm{g} / \mathrm{mL})$. The vertical line in each plot at 2 min marked the introduction of PMB. The optical images of each cell at the end of the experiment are shown at the bottom of each response plot (Scale bar: $500 \mathrm{~nm}$ ). 
All recorded images were used for analysis, and all displacement plots were smoothed by averaging 20 frames.

\section{Results and Discussion}

\subsection{Detection Limit}

To estimate the detection limit of the method, we performed experiments on dust particles and $1-\mu \mathrm{m}$ silicon beads on the glass slide (Fig. 4). We estimated a detection limit of $\sim 9 \mathrm{~nm}$ (defined as three times the standard deviation). We also tested and measured the system's response to drift changes in the focus plane (Fig. 5).

\subsection{Polymyxin B Results}

Using the optical tracking technique described in the previous section, we obtained the local displacement vectors of an $E$. coli
O157:H7 cell before and after exposure to PMB from which we determined the corresponding length changes of the bacterial cell [Fig. 6(a)]. Before exposure to PMB, the bacterial cell incubated in $1 \times$ PBS buffer exhibited minimal changes in cell length [blue curve, Fig. 6(a)]. Upon introducing PMB, cell length initially expanded by $\sim 40 \mathrm{~nm}$ [arrow, Fig. 6(a)], followed by a large ( 200 nm) shrinkage [red curve, Fig. 6(a)]. The PMB antibiotic is a cationic peptide, which elicits bactericidal activity against most gram-negative bacilli, including E. coli $\mathrm{O} 157: \mathrm{H} 7{ }^{18}$ The observed PMB-induced bacterial cell deformation arises because PMB binds to the negatively charged lipopolysaccharide layer of the cell and destabilizes the bacterial outer membrane, ${ }^{18}$ as the fatty acid portion of PMB dissolves in hydrophobic region of cytoplasmic membrane and disrupts the membrane integrity. ${ }^{19}$ Next, we measured PMB-induced membrane deformation (edge displacement via length and width changes) in 40 E. coli $\mathrm{O} 157: \mathrm{H} 7$ cells exposed to a high
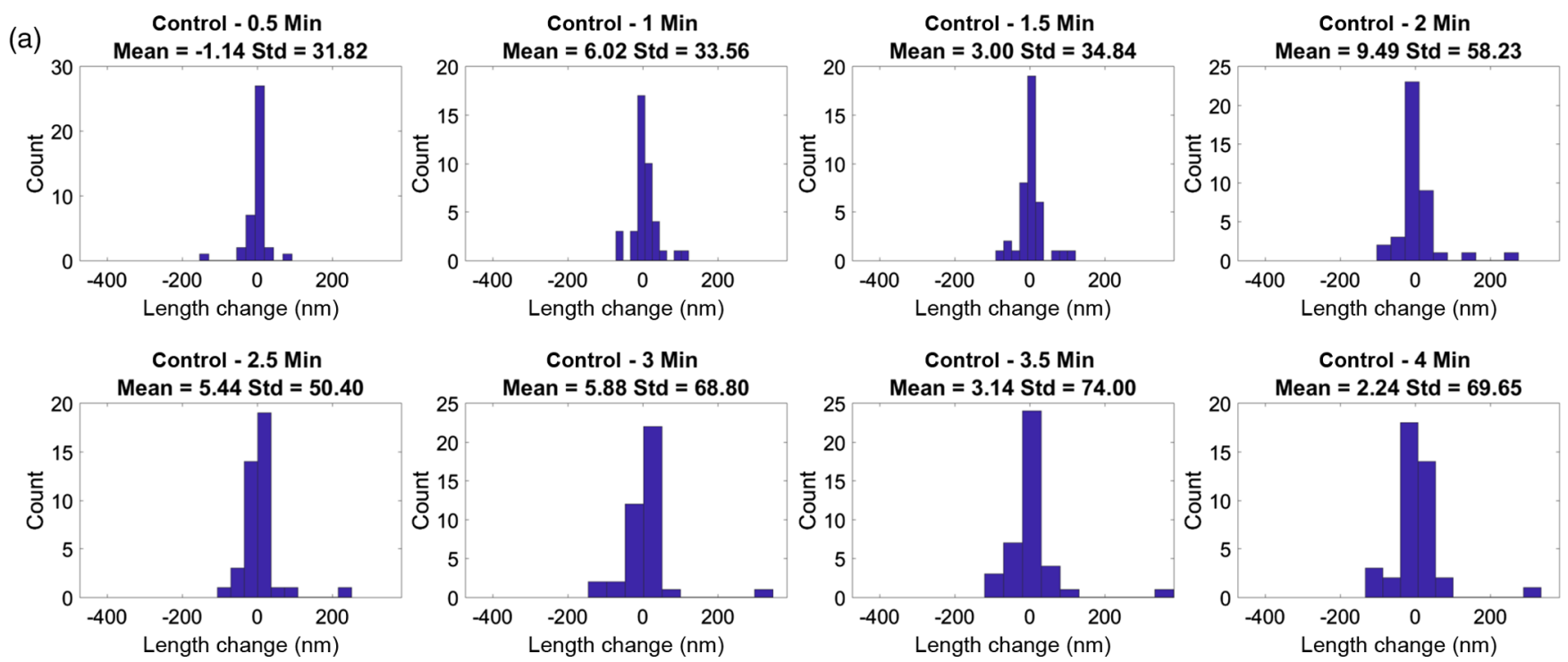

(b)
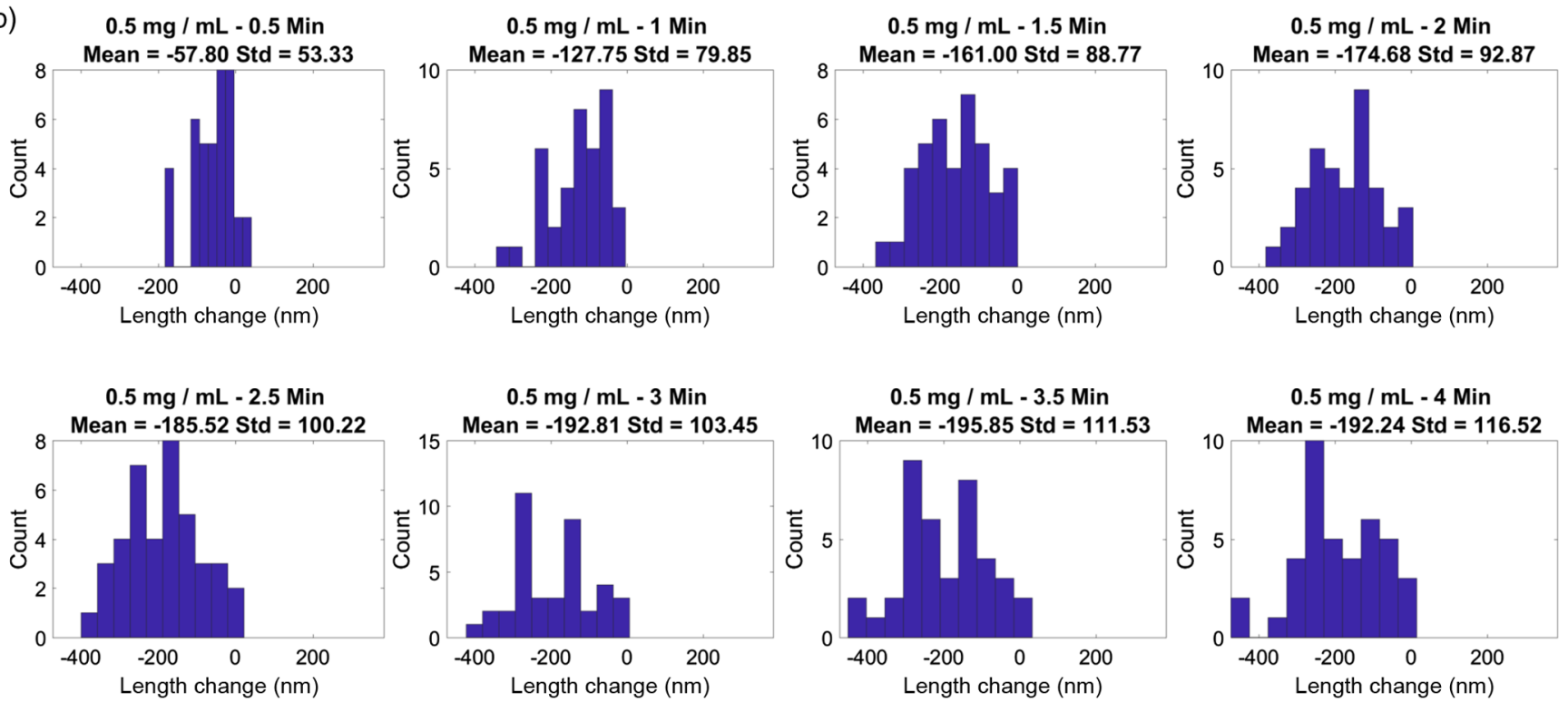

Fig. 8 Histogram analyses of length changes for 40 cells in PBS control (a) and PMB (b) experiments at different time points (after introduction of antibiotics for PMB plots). After antibiotic addition, cell shrinkage increased over time. Comparison of average values between the control and antibiotic experiments for all time points revealed statistically significant differences $\left(p=3.43 \times 10^{-8}\right.$; Student $t$-test). 
concentration of PMB $(0.5 \mathrm{mg} / \mathrm{mL})$ [Figs. 6(b) and 7]. Length and width changes were calculated by summing opposite displacement vectors, thereby excluding effects caused by changes in cell motion. We intentionally chose a high bactericidal PMB antibiotic concentration, significantly higher than the determined PMB minimum inhibitory concentration (MIC) of $8 \mu \mathrm{g} / \mathrm{mL}$, to quickly observe deformation in most of the cells, as opposed to using low concentrations where only a few cells showed a significant response. Nearly all cells showed a small, initial expansion in length, followed by a significant decrease in length, which was captured within seconds after PMB addition. Width change in individual cells was less obvious with a few cells displaying decreased width while other cells increased in width. Although there was also variability in the total length decreases between cells, these variations are expected, given the phenotypic heterogeneity of bacterial cells. $^{20}$ Bacterial heterogeneity and diversified phenotypic responsiveness underscore the importance of analyzing individual cells with the imaging-based method. Detailed statistical analysis and histograms are shown in Figs. 8 and 9. Compared with traditional AST methods that measure an averaged feature (e.g., turbidity) of the entire sample, single cell analysis could identify a few antibiotic resistant cells among a large population of susceptible cells by tracking the phenotypic responses of the resistant cells to the antibiotic, which could lead to faster AST.

Although the detection limit of the method itself is very small $(\sim 9 \mathrm{~nm})$, external factors, such as the microscope stability (Fig. 3) and the attachment of the bacterial cells to the surface, contribute to the accuracy of the measurement. Microscope focus drift can lead to systematic errors in the measurement of the membrane deformation. Micromotion of cells weakly attached to the surface may also affect the deformation tracking, especially when the cell moves out of the focal plane. Moreover,
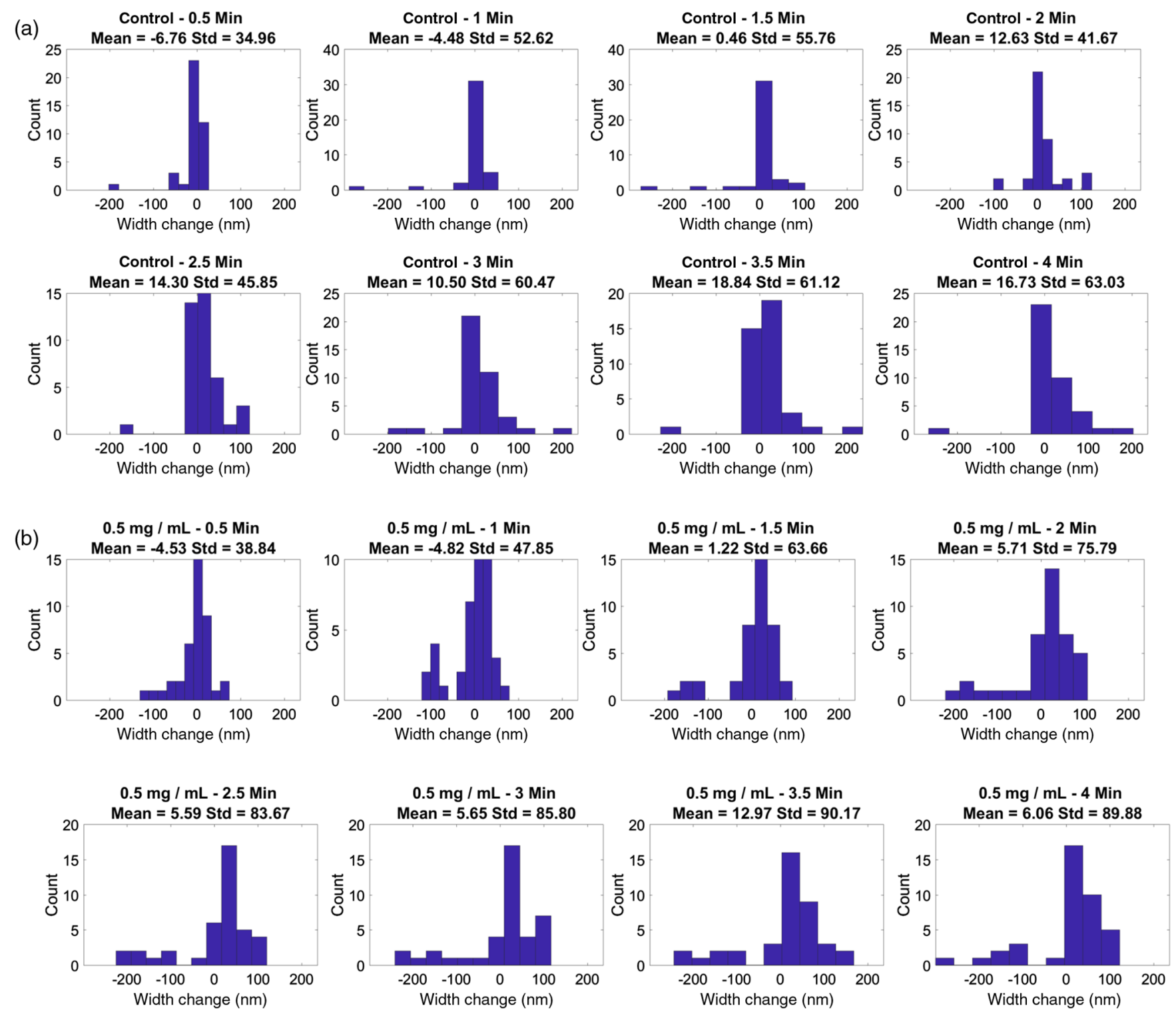

Fig. 9 Histogram analyses of width changes for 40 cells in PBS control (a) and PMB (b) experiments at different time points (after introduction of antibiotics for PMB plots). After antibiotic addition, cell width did not significantly change over time. Comparison of average values between the control and antibiotic experiments for all time points revealed no statistical differences ( $p=0.75$; Student $t$-test). 
cells in the solution may occasionally move on top of a cell on the surface, thereby blocking the surface-immobilized cell from the view of the microscope. These external factors may be reduced by stabilizing the microscope and developing improved cell attachment methods.

\subsection{Ampicillin Results}

We have also studied UPEC CFT073 exposed to the antibiotic, ampicillin, ${ }^{21,22}$ and analyzed the local cellular deformation of individual cells (Fig. 10). Unlike PMB-treated E. coli O157: $\mathrm{H} 7$, ampicillin causes the UPEC cell to bulge and elongate until cell lysis. ${ }^{22}$ Ampicillin targets enzymes that are critical

(a)

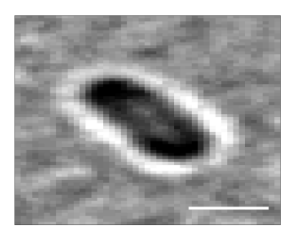

$T=0 \min$ (b)

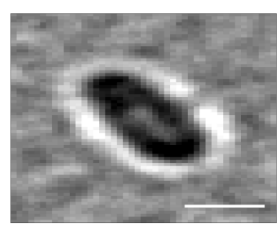

Fig. 10 Membrane deformation of a UPEC cell exposed to ampicillin (1 mg/ml). (a) Normal cell, (b) beginning of bulge formation, (c) large bulge, and (d) cell lysis. Scale bar: $200 \mathrm{~nm}$.

(a)

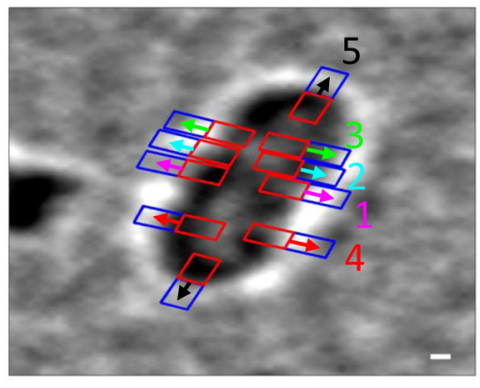

(c)

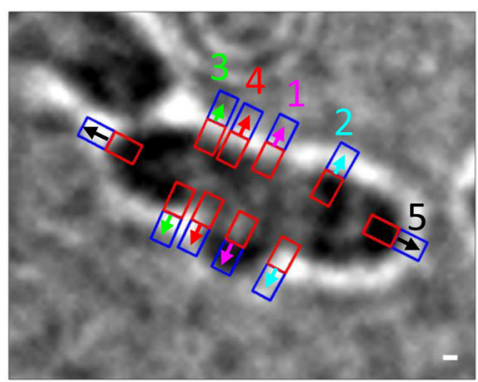

(e)

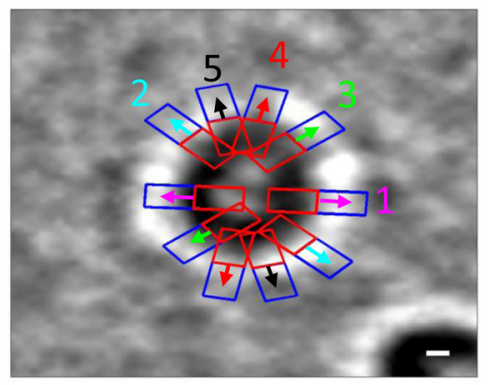

in forming bacterial peptidoglycan, a thin cell wall layer in gram-negative bacteria composed of long glycan chains, which serve to maintain cell structure and balance turgor pressure. ${ }^{23}$ By inhibiting peptidoglycan transpeptidases, ampicillin inhibits cell wall synthesis and bacterial cell division. ${ }^{21,24}$ Therefore, ampicillin causes the cells to bulge, producing a cytosolic protrusion surrounded by both the cytoplasmic and outer membranes, near the cell septum, and induces expression of autolysins, subsequently leading to cell lysis. ${ }^{22}$ Figure 10 shows different stages of a UPEC cell exposed to ampicillin, with an initial normal appearance, an initial membrane-bound cytoplasmic bulge formed within a few minutes, followed by a large bulge and subsequent lysis. ${ }^{24}$

(c)

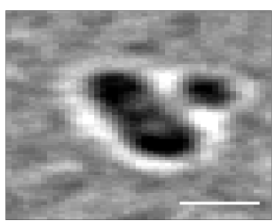

$T=35 \mathrm{~min}$ (d)

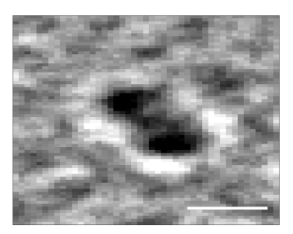

$T=37 \mathrm{~min}$

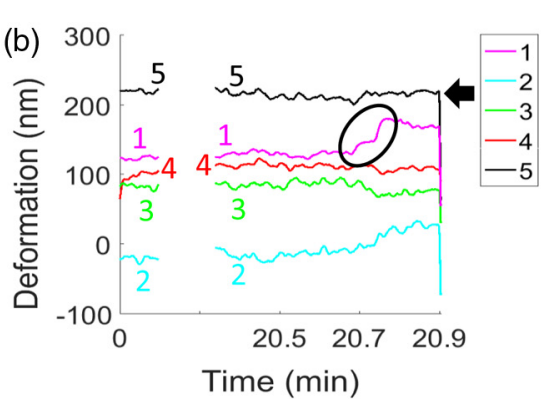

(d) 150
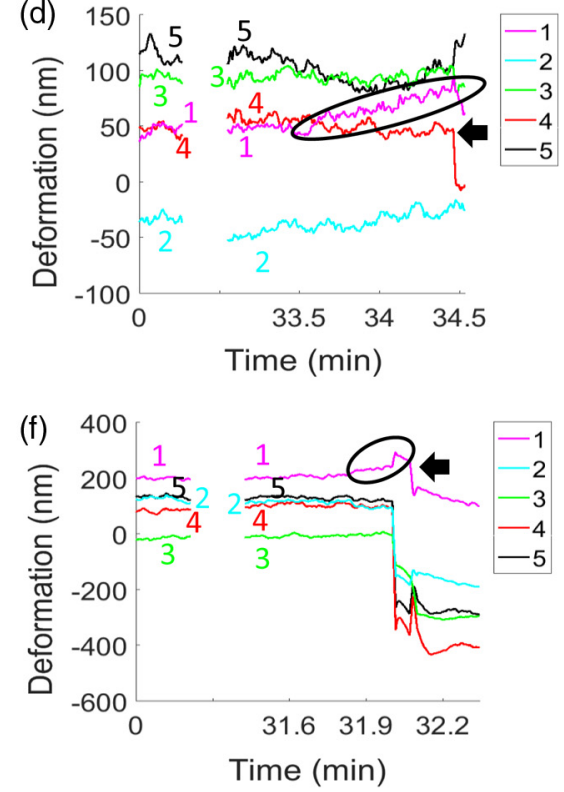

Fig. 11 Displacement measurements of UPEC cells exposed to ampicillin $(1 \mathrm{mg} / \mathrm{ml})$. Displacement vectors $(a, c$, and $e)$ and corresponding cell plots $(b, d$, and $f)$ are shown for different regions of different cells. The bulge region is shown with magenta vectors and marked as " 1 ". The circled regions (b, $d$, and f) indicate the bulge membrane deformation while the arrows indicate cell lysis. Scale bar 200: $\mathrm{nm}$. 
Figure 11(a) shows the image of a UPEC cell captured $20 \mathrm{~min}$ after exposure to a high concentration of ampicillin $(1 \mathrm{mg} / \mathrm{mL})$. Similar to the PMB experiments, we exposed cells to an ampicillin concentration significantly higher than the determined MIC $(4 \mu \mathrm{g} / \mathrm{mL})$ to rapidly induce cellular responses. The local displacements associated with cell membrane deformation at different locations reveal detailed time characteristics linking the formation of a bulge to cell lysis [Fig. 11(b)]. The bulge region (magenta, and marked by "1") gradually increases the displacement as the protrusion forms (marked by a circle in the plot). A second location (marked by "2") next to region "1" also shows a displacement increase (cyan curve), indicating that the observed displacement increase in location " 1 " is not due to noise. The displacements in other locations (e.g., regions 3 to 5) of the cell display minimal changes. Although the initial bulge formation is small, it can be resolved within $\sim 9 \mathrm{~nm}$, demonstrating the advantage of the present method over other optical imaging and cell segmentation approaches. ${ }^{24}$ After the cellular bulge expands over time, cell expansion slows and is followed by a sudden decrease as cell lysis initiates (marked by thick black arrows). This characteristic cellular response was observed in other UPEC cells [Figs. 11(c)-11(f)], but the magnitude and initiation of cellular deformation vary due to heterogeneity of different cells. $^{24}$

We have demonstrated a sensitive optical method to track nanometer-scale cell deformation and growth as a phenotypic feature for potential AST using two antibiotics with distinct action mechanisms on the cell membrane and cell wall, PMB and Ampicillin, as examples. Other antibiotics with different mechanisms may not have the same rapid effects on cell deformation. To further develop it into technology for AST, substantial validation experiments will be needed. Examples include testing of other common antibiotics over clinically relevant concentration ranges, both susceptible and resistant strains, and also real clinical samples.

\section{Conclusion}

We have developed an optical imaging method to track antibiotic activity on individual bacterial cells in real-time. The method measures bacterial cellular deformations with $\sim 9-\mathrm{nm}$ detection limit, allowing sensitive detection of antibioticinduced changes in size, shape, and local membrane deformations of single bacterial cells. Using this capability, we have investigated the effects of $\mathrm{PMB}$ on E. coli $\mathrm{O} 157: \mathrm{H} 7$ and ampicillin on UPEC. PMB caused E. coli O157:H7 to shrink along the cell edge, whereas ampicillin caused the cellular bulge to form in UPEC. Based on tracking membrane deformation changes of single cells, our method will help identify individual cells in a sample that are susceptible to antibiotics in real-time. The current detection limit is mainly determined by the stability of the microscope and the attachment of the bacterial cells to the surface. This detection limit may be improved for more accurate tracking of antibiotic-induced bacterial membrane deformations, which may lead to a sensitive AST method. The present work focused on developing a sensitive optical tracking method to detect cell deformation associated with antibiotic activity. Further validation experiments must be performed with additional antibiotics and bacteria, including antibiotic-resistant strains, to establish MIC in conjunction with traditional AST methods.

\section{Disclosures}

The authors have no relevant financial interests in this article and no potential conflicts of interest to disclose.

\section{Acknowledgments}

We thank Prof. T.C. Li for helpful discussions, and China Natural Science Foundation (\#2137008 and \#2137902) and Gordon and Betty Moore Foundation for financial support.

\section{References}

1. T. Frieden, Antibiotic Resistance Threats in the United States, pp. 22 50, CDC (2013).

2. J. O'Neill, "Antimicrobial resistance: tackling a crisis for the health and wealth of nations," The Review on Antimicrobial Resistance, pp. 1-16 (2014).

3. Z. Golkar, O. Bagasra, and D. G. Pace, "Bacteriophage therapy: a potential solution for the antibiotic resistance crisis," J. Infect. Dev. Countries 8(2), 129-136 (2014).

4. J. G. Bartlett, D. N. Gilbert, and B. Spellberg, "Seven ways to preserve the miracle of antibiotics," Clin. Infect. Dis. 56(10), 14451450 (2013).

5. K. Syal et al., "Current and emerging techniques for antibiotic susceptibility tests," Theranostics 7(7), 1795-1805 (2017).

6. J. Muscedere et al., "Comprehensive evidence-based clinical practice guidelines for ventilator-associated pneumonia: diagnosis and treatment," J. Crit. Care 23(1), 138-147 (2008).

7. C. Liu et al., "Clinical practice guidelines by the Infectious Diseases Society of America for the treatment of methicillin-resistant Staphylococcus aureus infections in adults and children," Clin. Infect. Dis. 52(3), e18-e55 (2011).

8. A. Dufour et al., "Signal processing challenges in quantitative 3-D cell morphology," IEEE Signal Process. Mag. 32, 30-40 (2015).

9. K. Syal et al., "Antimicrobial susceptibility test with plasmonic imaging and tracking of single bacterial motions on nanometer scale," ACS Nano 10(1), 845-852 (2015).

10. I. Sinn et al., "Asynchronous magnetic bead rotation microviscometer for rapid, sensitive, and label-free studies of bacterial growth and drug sensitivity," Anal. Chem. 84(12), 5250-5256 (2012).

11. G. Longo et al., "Rapid detection of bacterial resistance to antibiotics using AFM cantilevers as nanomechanical sensors," Nat. Nanotechnol. 8(7), 522-526 (2013).

12. Y.-L. Chiang et al., "Innovative antimicrobial susceptibility testing method using surface plasmon resonance," Biosens. Bioelectron. 24(7), 1905-1910 (2009).

13. J. Karasinski et al., "Detection and identification of bacteria using antibiotic susceptibility and a multi-array electrochemical sensor with pattern recognition," Biosens. Bioelectron. 22(11), 2643-2649 (2007).

14. J. Choi et al., "A rapid antimicrobial susceptibility test based on singlecell morphological analysis," Sci. Transl. Med. 6(267), 267ra174 (2014).

15. C. S. Price, S. E. Kon, and S. Metzger, "Rapid antibiotic susceptibility phenotypic characterization of Staphylococcus aureus using automated microscopy of small numbers of cells," J. Microbiol. Methods 98(1), 50-58 (2014).

16. J.-V. Holtje, "From growth to autolysis: themurein hydrolysis in Escherichia coli," Arch. Microbiol. 164, 243-254 (1995).

17. Y. Guan et al., "Detection of molecular binding via charge-induced mechanical response of optical fibers," Sci. Adv. 5, 4375-4381 (2014).

18. R. Daugelavičius, E. Bakienè, and D. H. Bamford, "Stages of polymyxin B interaction with the Escherichia coli cell envelope," Antimicrob. Agents Chemother. 44(11), 2969-2978 (2000).

19. M. Krupovič, R. Daugelavičius, and D. H. Bamford, "Polymyxin B induces lysis of marine pseudoalteromonads," Antimicrob. Agents Chemother. 51(11), 3908-3914 (2007).

20. M. Zimmermann et al., "Phenotypic heterogeneity in metabolic traits among single cells of a rare bacterial species in its natural environment quantified with a combination of flow cell sorting and NanoSIMS," Front. Microbiol. 6, 1-11 (2015). 
21. B. G. Spratt, "Distinct penicillin binding proteins involved in the division, elongation, and shape of Escherichia coli K12," Proc. Natl. Acad. Sci. U.S.A. 72(8), 2999-3003 (1975).

22. I. D. J. Burdett and R. G. E. Murray, "Electron microscope study of septum formation in Escherichia coli strains B and B/r during synchronous growth," J. Bacteriol. 119(3), 1039-1056 (1974).

23. J. V. Holtje, "Growth of the stress-bearing and shape-maintaining murein sacculus of Escherichia coli," Microbiol. Mol. Biol. Rev. 62(1), 181-203 (1998).

24. Z. Yao, D. Kahne, and R. Kishony, "Distinct single-cell morphological dynamics under beta-lactam antibiotics," Mol. Cell 48(5), 705-712 (2012).

Rafael Iriya is an alumni of the School of Electrical, Computer and Energy Engineering at Arizona State University. He received his $B S$ and MS degrees in electrical engineering from the University of Sao Paulo, Brazil. This project is part of his PhD thesis. His interests include signal processing and machine learning for image and audio applications.

Shelley E. Haydel is an associate professor in the ASU School of Life Sciences and Biodesign Institute Center for Immunotherapy, Vaccines and Virotherapy and has expertise in medical microbiology, infectious diseases, and diagnostics. Her research laboratory is engaged in numerous interdisciplinary projects involving tuberculosis, antibiotic-resistant bacterial infections, novel antibacterials, integrative treatments for skin and soft tissue bacterial infections, rapid antibiotic susceptibility testing platforms, infectious disease biomarkers, and novel bioactive skin sealants.

Shaopeng Wang is a research professor in the Biodesign Center for Bioelectronics and Biosensors at Arizona State University. His research interest is in the development of biosensors and bioinstrumentations. He received his BS degree in biology and his MS degree in biophysics from Tsinghua University. He received his $\mathrm{PhD}$ in physical chemistry from the University of Miami. He has 100 peer-reviewed publications, book chapters, patents and conference proceedings, with $4000+$ citations and an h-index of 31 .

Nongjian Tao joined the ASU faculty as a professor of electrical engineering and an affiliated professor of chemistry in 2001. He is currently serving as the director of the Biodesign Center for Bioelectronics and Biosensors at ASU and an associate editor for ACS Sensors. He is elected fellows of AAAS, APS and AIMBE. His current research interest includes chemical and biological imaging and sensing technologies, molecular electronics, and wireless sensors for mobile health applications.

Biographies for the other authors are not available. 